Pecvnia, 7 (2008), pp. 285-311

\title{
Encontrando al Kaizen: Un análisis teórico de la Mejora Continua
}

Recibido: Enero 2009 Aceptado: Mayo 2009

\author{
Manuel F. Suárez-Barraza \\ manuelfrancisco.suarez@itesm.mx \\ Escuela de Graduados en Administración y \\ Dirección de Empresas EGADE Zona Centro, \\ Tecnológico de Monterrey \\ Av. Las Torres $\mathrm{s} / \mathrm{n}$ casi esq. con Av. Tecnológico, San Salvador \\ Tizatlalli, Metepec, Edo. de México, C.P. 52172 (México) \\ José-Á. Miguel-Dávila \\ jam.davila@unileon.es \\ Universidad de León \\ Fac. de Ciencias Económicas y Empresariales \\ Campus de Vegazana, $\mathrm{s} / \mathrm{n}$ \\ 24071 León (España)
}

Desde que Masaaki Imai acuño el término de Kaizen a mediados de los años ochenta, este se ha visto como un elemento clave para la competitividad de las empresas japonesas. No obstante, y a pesar de que el Kaizen ha sido definido por el autor que condujo a la luz el término, todavía sigue existiendo en la literatura sobre el tema cierta ambigüedad e inconsistencia. Además, existe una clara necesidad de desarrollar esta teoría en el campo de la dirección de operaciones.
Since Masaaki Imai coined the term Kaizen in the mid eighties it has been regarded as a key element in the competitiveness of Japanese companies. However, even though Kaizen was defined by the author who created the term, writings by scholars and practitioners in the field exhibit a certain degree of ambiguity and inconsistency. Finally, there is a clear need to develop this theory in the field of operations management.

The purpose of this paper is to analyze 
El propósito de este trabajo es analizar el Kaizen en la literatura académica y práctica con el fin de mejorar, explorar y hacer una contribución a su potencial perfil teórico. Se realizó una revisión de la literatura usando Kaizen como término de búsqueda en varias bases de datos: Proquest, Business Source Elite, Business Source Premier, Emerald, Science Direct and Google Scholar. También se consultaron libros escritos tanto por académicos como por los que lo practican. La literatura concerniente al Kaizen fue metodológicamente analizada y clasificada. Los hallazgos del estudio indican que el Kaizen es presentado bajo tres perspectivas o esferas, que incluyen una serie de principios y técnicas. Comparando los tres puntos de vista, emergieron un conjunto de principios $\mathrm{y} / \mathrm{o}$ piedras angulares.

Palabras clave: Mejora continua, Kaizen, Análisis conceptual, Revisión de artículos.

\begin{abstract}
Kaizen in the academic and practitioner literature in order to better understand it and further explore and contribute to its potential theoretical profile. A literature review was carried out using Kaizen as a search term. Various databases were used for this purpose: Proquest, Business Source Elite, Business Source Premier, Emerald, Science Direct and Google Scholar. Books written by both scholars and by practitioners on the subject were also consulted. The literature concerning Kaizen was methodically analyzed and categorized. The findings of the study indicate that Kaizen is presently displayed under three perspectives or spheres, which include a series of principles and techniques. By comparing the three viewpoints, a set of guiding principles and/or cornerstones for Kaizen have also emerged.
\end{abstract}

Key words: Continuous Improvement, Kaizen, Conceptual Analysis, Review Articles, Guiding Principles of Kaizen.

\section{INTRODUCCIÓN}

Desde que Masaaki Imai acuñara e introdujera el término Kaizen a mediados de los años ochenta en su libro el KAIZEN - The key to Japan's Competitive Success (1986), en términos de una posible definición, de sus características y su relación con las prácticas gerenciales japonesas, el término Kaizen entró en la arena del management, como el posible "elemento perdido" del éxito operacional de las empresas japonesas. De hecho, el concepto cobró tanta fuerza en este sentido, que el Kaizen ha sido considerado como un elemento clave para la competitividad de las organizaciones japonesas en los últimas tres décadas del siglo XX (Imai 1986; Brunet 2000). Durante los últimos 20 años, este concepto ha sido utilizado en el mundo empresarial de diferentes maneras: i) desde aplicarlo como un posible elemento "mágico" que aparece a través de la participación de los empleados y los sistemas de sugerencias de mejora, considerándose de igual manera, como un grupo de técnicas y herramientas para eliminar desperdicios (Muda por su término en japonés); ii) hasta llegar a ser simplemente utilizado como una pieza más, de aproximaciones de gestión tales como la Gestión por Calidad Total - Total Quality Management (TQM)en su enfoque occidental, la Gestión de la Calidad en toda la Empresa 
- Company Wide Quality Control (CWQC) - en su orientación japonesa, el Pensamiento Esbelto - Lean Thinking- o el Sistema de Producción Toyota (Martínez-Lorente et al. 1999; Osono et al. 2008). No obstante, y a pesar de que el Kaizen ha sido definido por el autor que condujo a la luz el término, todavía sigue existiendo en la literatura del tema, cierta ambigüedad e inconsistencia en la forma en que el Kaizen aparece en la literatura académica y en la empresarial. Por ende, el desarrollo de teoría en este campo de la gestión de operaciones, surge como necesario.

Ante todo este contexto, en este artículo se analiza como primer aspecto, las posibles definiciones del Kaizen en la literatura académica existente, para posteriormente, analizar las investigaciones que se han realizado sobre el mismo, tanto en la literatura académica como en la gerencial y de divulgación del tema. De los dos aspectos anteriores, se desprenden los elementos necesarios para las contribuciones y conclusiones de nuestro análisis teórico. Como resultado de lo anterior, se analiza y se muestra nuestra propia contribución al tema, describiéndolo y organizándolo en forma de tres diferentes esferas concéntricas por las cuales el Kaizen se puede presentar. Se finaliza con las conclusiones del estudio, en los cuales se muestran ciertos aspectos, que pueden ser considerados para seguir siendo analizados, desarrollados o mejorados; entre ellos, un esquema conceptual que interrelaciona las tres esferas propuestas. Todo ello, con el fin de generar una mayor comprensión del Kaizen, y quizás, conformar una futura guía de investigación tanto teórica como práctica.

\section{REVISIÓN DE LA LITERATURA}

\section{1. ¿Qué es el Kaizen?}

A pesar de que el término Kaizen es definido por Masaaki Imai en sus dos libros del tema (1986; 1997), esta palabra japonesa que significa «mejoramiento», todavía no tiene una explicación detallada que le permita brindar mayor claridad de su contenido teórico. Diferentes autores ha intentado explicarlo desde diferentes perspectivas. El propio Imai (1989: 23) lo define como: "Mejoramiento y aún más significa mejoramiento continuo que involucra a todos, gerente y trabajadores por igual". Para Newitt (1996), la definición de Imai (1986, 1989), se basa en que la palabra Kaizen es una derivación de dos ideogramas japonesas que significan: $K A I=$ Cambio, ZEN = Bueno (para mejorar) (Farley 1999; Newitt 
1996), es decir, Mejora Continua o Principio de Mejora Continua (Lillrank y Kano 1989: 28).

De esta manera, la primera óptica de la definición del Kaizen se basa entonces en sustentar su presencia, como un elemento organizacional en la que la participación de los empleados impacta directamente en la mejora de los procesos de trabajo (Senge 1990; Elgar y Smith 1994). En este sentido, Bessant (2003) indica que la movilización y la participación de los empleados, genera un canal o un medio para que los mismos, puedan contribuir al desarrollo de la compañía. En términos sencillos, "trabajando con las manos, pero utilizando el cerebro para pensar". Esta idea, se compara y es similar a los estudios iniciales de la Escuela de las Relaciones Humanas, en las que Mayo, Maslow, McGregor y Herzberg argumentaban dicha aproximación del management (Malloch 1997).

En este sentido, la Asociación de Relaciones Humanas del Japón (1992: 4) señala que para los japoneses, la palabra Kaizen se asume como un símbolo a los problemas y luchas de cada día, y del modo en el que los empleados se enfrentan a todo ello. De hecho, en ocasiones el Kaizen ha sido visto como una fuerza ética (interna que se encuentra en uno mismo) de cada trabajador, que es capaz de resolver problemas en el día a día, plenamente convencido y de manera voluntaria (Styrhe 2001). Por ello ciertos autores, comienzan a verlo como una «filosofía de vida», en la que se pueden abarcar los ámbitos personales, familiares, sociales y por supuesto la vertiente del trabajo (Imai 1986; Wittenberg 1994; Gondhalekar et al. 1995). Debido a esta tendencia de entender al Kaizen, como un principio armonizador del entorno con los valores de cada individuo, otros autores incluso, establecen su origen en la filosofía confusionista, la cual establece un profundo respeto y armonía por el medio ambiente, a través de un equilibrio entre el individuo y la naturaleza (Styhre 2001; Bodek 2002; Imai 2006). Por este motivo es visto como un principio o «espíritu individual» de Cooperación y Mejora (Brunet 2000: 2 ), que rápidamente se despliega generando un impacto positivo en la sociedad (Gondhalekar et al. 1995: 197).

Por otro lado, en una investigación específica del tema en organizaciones japonesas, se observó la amplia variedad que existe acerca de cómo se comprende y se aplica el Kaizen. De hecho, dicha comprensión y aplicación fue acorde a las características de cada organización estudiada. Brunet y New (2003: 1428) autores de esta investigación, definen al Kaizen como: "Un mecanismo penetrante de actividades continuas, donde las personas involucradas juegan un rol explícito, para identificar y asegurar 
impactos o mejoras que contribuyen a las metas organizacionales". Recientemente, el más conocido promotor del término y acuñador del mismo, Masaaki Imai (2006; 2007), indicaba que el Kaizen significa:

mejoramiento continuo, pero mejoramiento todos los días, a cada momento, realizado por todos los empleados de la organización, en cualquier lugar de la empresa. Y que va de pequeñas mejoras incrementales a innovaciones drásticas y radicales.

En ese mismo orden de ideas, otros autores que siguen la misma línea de enseñanzas de Taichi Ohno en Toyota Motor Company, van más allá, e indican que el mejoramiento del Kaizen por parte de los empleados, debe llegar a hacerse incluso, cada segundo de su tiempo, aunque sea en pensamiento (Takeyuki 1995; Suárez-Barraza 2007). Mientras, el JUSE (Japanese Union for Scientifist and Engineers), utiliza el término Kaizen sin concretarlo, como un axioma para definir otros conceptos y como una filosofía empresarial (Bhuiyan y Baghel 2005). Así pues, Suárez-Barraza (2007: 91) lo define como:
Una filosofía de gestión que genera cambios o pequeñas mejoras incrementales en el método de trabajo (o procesos de trabajo) que permite reducir despilfarros y por consecuencia mejorar el rendimiento del trabajo, llevando a la organización a una espiral de innovación incremental.

Finalmente, también se encuentra la línea de autores que indican que el Kaizen o la Mejora Continua en su caso, puede ser entendido, como un elemento más de la Gestión por Calidad Total (Crosby 1979; Ishikawa 1986; Deming 1986; Garvin 1987; Juran 1990; Hakes 1991; Feigenbaum 1991; Dean y Bowen 1994; Oakland 1999; Hellsten y Klefsjö 2000), o como la plataforma básica de Sistema de Producción Toyota o el Pensamiento Esbelto (o Lean Thinking) caracterizado por la participación de los empleados en la solución de los problemas o desperdicios (Muda) que surgen en el trabajo cotidiano; la forma en que se ejecuta dicha eliminación es a través de equipos de mejora o de la aplicación de las 5'S y la estandarización (Womack et al. 1990; Ho and Citmill 1996; Likert 2004; Spear 2004; Hino 2006; Dahlgaard y Dahlgaard-Park 2006). Ambas aproximaciones, la japonesa y la occidental también ha sido estudiada por algunos autores para comparar conceptos, similitudes y principios (Magaña-Campos y Aspinwall 2003). 
A manera de resumen, es importante indicar después de analizar la literatura que el Kaizen ha sido un término que sigue en evolución, lo que ha traído como consecuencia, diferentes significados dependiendo del tiempo y el contexto organizacional en que se ha presentado (Tozawa y Bodek 2002).

\subsection{Análisis del Kaizen en la literatura existente}

Se realizó una revisión de la literatura a partir de utilizar como término de búsqueda la palabra Kaizen, utilizando para ello diferentes bases de datos: Proquest, Business Source Elite, Business Source Premier, Emerald, Science Direct y Google Scholar. De manera adicional, también se abordaron libros tanto de corte académico como gerencial divulgativo que estuvieran relacionados con el tema. Como resultado de esta exploración, se encontró que el Kaizen en la literatura existente, aparece referido en dos tipos de estudios principalmente. Aquéllos que investigan al Kaizen en su orientación japonesa, referidos a la definición de Imai (1986) y aquéllos que se refieren al Kaizen, en su definición occidental, como «Mejora Continua (MC)».

\subsubsection{El Kaizen en su orientación japonesa}

En primer lugar, la literatura que se refiere al Kaizen en su orientación japonesa es escasa desde un ángulo académico. No obstante, durante la búsqueda se pudieron encontrar varios artículos del mismo, circunscrita a casos o ejemplos de orientación práctica. Referido a lo anterior, en un artículo publicado en la Revista Strategic Direction (2004: 23) se indica que las investigaciones acerca del Kaizen, en su orientación japonesa, siguen siendo aisladas, por no decir, nulas. Sólo hacen referencia al trabajo de Brunet y New (2003) de la Said Business School en Oxford. Aún así, una referencia básica del término Kaizen en la literatura se refiere a su posible origen en los valores subyacentes de la empresa Toyota Motor Company (Nemoto 1987). Para Ohno (1978: 42), el fundador del Sistema de Producción Toyota la mejora continua o Kaizen se entendía como que "la mejora tiene dos vertientes principales: es eterna e infinita". Esto reafirma que el Kaizen no es un programa de mejora aislado, sino que por el contrario es considerado en dicha organización como un elemento filosófico de su modelo de negocio (el Toyota Way 2001) (Osono et al. 2008). De hecho, en la actualidad, de acuerdo al 
periódico Nikkei Sangyo muchas compañías japonesas siguen la tendencia de Toyota Motor Company por tener en su modelo de negocios al Kaizen (Nikkei Sangyo Shimbum 2007).

Entrando más específicamente en la literatura, dentro de los estudios del Kaizen en su orientación japonesa se encuentra la investigación cuantitativa que presenta Cheser (1998) acerca del efecto que produce el Kaizen en la motivación de los empleados en plantas de manufactura japonesa. Sus resultados indican que la motivación de los empleados en estas organizaciones se incrementó en los primeros 6 meses como resultado de la aplicación del Kaizen en forma del Sistema de Producción Toyota en las plantas de manufactura analizadas. Se generaban con ello un alto nivel de cambio positivo en la motivación de los empleados (correlación positiva). Concluye indicando el área de oportunidad que existe por estudiar el Kaizen desde un punto de vista académico (Cheser 1998: 213). Otro estudio empírico similar, es el ya señalado de Brunet y New (2003); en su investigación de 11 empresas japonesas de acero y de automóviles (Nippon Steel, Honda, Susuki, Toyota Kyushu, Sumitomo Metal, etc.), concluyen que existe una considerable diversidad en la forma en que el Kaizen opera en las organizaciones japonesas. Pero al mismo tiempo, estos autores también indican, que existen profundas similitudes, sobre todo en la integración del rol del Kaizen en el sistema de gestión y en sus principios básicos (core principles) al momento de su implementación en las organizaciones japonesas (Brunet y New 2003). En una línea similar, Aoki (2008) concluye que es factible transferir el Kaizen a otros países que cuentan con una cultura diferente a la japonesa, siempre y cuando se logren implementar en dichas organizaciones fuera del Japón las capacidades fundamentales del Kaizen (sus principios básicos) que son, desde la óptica del autor: la propia y auto iniciativa de los trabajadores por buscar mejoras; la comunicación inter-departamental y funcional; la disciplina de los propios empleados; y la estandarización del trabajo con el objetivo de eliminar el Muda.

En otros artículos más conceptuales acerca del Kaizen, se encuentra la argumentación de Cheser (1994), al indicar que el Kaizen va más allá de la forma con la que se define en occidente, es decir, de la Mejora Continua. En otros términos, para Cheser (1994) el Kaizen se diferencia de la Mejora Continua en los propios principios rectores que lo delimitan. Y aunque Imai (1986, 1989), no se refiere directamente a un grupo de principios rectores, indica que el Kaizen puede ser delimitado en al menos tres tipos o formas: 1) el Kaizen management, 2) el Kaizen 
Tabla 1. Investigaciones del Kaizen en su vertiente japonesa

\begin{tabular}{|c|c|c|c|}
\hline Autores & $\begin{array}{c}\text { Enfoque del Estudio / } \\
\text { Nivel de Análisis } \\
\end{array}$ & $\begin{array}{c}\text { Principales Conclusiones } \\
\text { de la Investigación }\end{array}$ & $\begin{array}{c}\text { Técnicas y Herramientas } \\
\text { del Kaizen utilizadas }\end{array}$ \\
\hline $\begin{array}{l}\text { Aoki } \\
(2008)\end{array}$ & $\begin{array}{l}\text { Empírico centrado en } \\
\text { el macro proceso de } \\
\text { construcción de } \\
\text { capacidades de la } \\
\text { organización }\end{array}$ & $\begin{array}{l}\text { Compuesto por cinco niveles evolutivos } \\
\text { que van del nivel precursor, de estructura, } \\
\text { estratégico, pro-activo, hasta alcanzar el } \\
\text { nivel de aprendizaje organizacional. }\end{array}$ & $\begin{array}{l}\text { - Estandarización } \\
\text { - Eliminación del muda } \\
\text { - Técnicas de comunicación } \\
\text { - Generación de disciplina } \\
\text { - Gestión inter-funcional (cross- } \\
\text { functional management) }\end{array}$ \\
\hline $\begin{array}{l}\text { Manos } \\
(2007)\end{array}$ & $\begin{array}{l}\text { Conceptual comparativo } \\
\text { entre el Kaizen y los } \\
\text { eventos Kaizen }\end{array}$ & $\begin{array}{l}\text { El Kaizen centrado en la vertiente japonesa } \\
\text { cuenta con cuatro características: } \\
\text { a) Pequeñas mejoras a lo largo del tiempo } \\
\text { b) Por medio de equipos de mejora } \\
\text { c) de bajo coste } \\
\text { d) se debe llevar incluso como una } \\
\text { forma de vida }\end{array}$ & $\begin{array}{l}\text { - Entrenamiento } \\
\text { - Eliminación del muda } \\
\text { - Estandarización } \\
\text { - } 5 \text { 'S } \\
\text { - Técnicas de solución de } \\
\text { problema } \\
\text { - Gestión Total del Flujo (Total } \\
\text { Flow Management) }\end{array}$ \\
\hline $\begin{array}{c}\text { Brunet y New } \\
(2003)\end{array}$ & $\begin{array}{l}\text { Empírico con } \\
\text { profundidad } \\
\text { longitudinal en } 11 \\
\text { empresas japonesas }\end{array}$ & $\begin{array}{l}\text { Se debe mantener las ideas o principios } \\
\text { rectores del Kaizen a pesar de la gran } \\
\text { variedad de prácticas, técnicas y } \\
\text { herramientas que se utilizan en las } \\
\text { organizaciones. }\end{array}$ & $\begin{array}{l}\text { - Despliegue de Políticas (Hoshin } \\
\text { Kanri) } \\
\text { - Concepto de Zero Defects } \\
\text { - Pequeños grupos de mejora } \\
\text { - Sistema de sugerencias }\end{array}$ \\
\hline $\begin{array}{l}\text { Berger } \\
(1997)\end{array}$ & $\begin{array}{l}\text { Conceptual orientado a } \\
\text { Principios Rectores }\end{array}$ & $\begin{array}{l}\text { Compuesto por tres principios rectores: } \\
\text { 1) el Kaizen orientado a los procesos; } \\
\text { 2) el Kaizen orientado al mantenimiento } \\
\text { y la mejora de los estándares; } \\
\text { 3) el Kaizen orientado a los individuos }\end{array}$ & $\begin{array}{l}\text { - Equipos de Mejora clasificados } \\
\text { en los siguientes apartados: } \\
\text { a) Círculos de Calidad } \\
\text { b) Equipos orgánicos de MC } \\
\text { c) Equipos de fuerza de tarea } \\
\text { d) Equipos de amplio enfoque } \\
\text { de la MC }\end{array}$ \\
\hline $\begin{array}{l}\text { Malloch } \\
(1997)\end{array}$ & $\begin{array}{l}\text { Empírico. Caso de } \\
\text { estudio en la empresa } \\
\text { UK Dieselco } \\
\text { (multinacional que } \\
\text { ensambla motores } \\
\text { diesel) }\end{array}$ & $\begin{array}{l}\text { La implementación del Kaizen requiere } \\
\text { una aproximación racional estratégica } \\
\text { por parte de los gerentes, para evitar } \\
\text { confusiones entre conceptos, técnicas y } \\
\text { herramientas por parte de los trabajadores. } \\
\text { Pero además añade, que la implementación } \\
\text { del Kaizen bajo el propio entendimiento } \\
\text { de los empleados, ayudó a Dieselco a } \\
\text { conseguir los objetivos de gestión } \\
\text { planteados }\end{array}$ & $\begin{array}{l}\text { - Equipos de mejora } \\
\text { - Estandarización } \\
\text { - Control Total de Calidad } \\
\text { - Mantenimiento Productivo } \\
\text { Total }\end{array}$ \\
\hline $\begin{array}{c}\text { Gondhalekar } \\
\text { et al. } \\
(1995)\end{array}$ & $\begin{array}{l}\text { Empírico. Caso de } \\
\text { estudio en la } \\
\text { organización India de } \\
\text { Godrej Soap Ltd. } \\
\text { Propone un modelo } \\
\text { teórico }\end{array}$ & $\begin{array}{l}\text { El Kaizen ayuda a la alineación entre los } \\
\text { objetivos de la empresa y la de los } \\
\text { empleados, y por lo tanto, dicha alineación } \\
\text { contribuye a su sostenibilidad a través de } \\
\text { una intervención activa de la gerencia } \\
\text { para mantener el esfuerzo }\end{array}$ & $\begin{array}{l}\text { - Sistemas de reconocimientos } \\
\text { - Formación activa } \\
\text { - Sistemas de sugerencias de } \\
\text { mejora } \\
\text { - Equipos de mejora }\end{array}$ \\
\hline $\begin{array}{l}\text { Tanner y } \\
\text { Roncarti } \\
(1994)\end{array}$ & $\begin{array}{l}\text { Empírico. Caso de } \\
\text { estudio en una } \\
\text { organización que lidera } \\
\text { el mercado de catéteres } \\
\text { intravenosos (Johnson } \\
\text { \& Johnson Medical's } \\
\text { Criticon Vascular } \\
\text { Access Facility) }\end{array}$ & $\begin{array}{l}\text { Los factores críticos cruciales para la } \\
\text { implementación del Kaizen son: } \\
\text { 1) enfoque en la acción: relacionado } \\
\text { con la puesta en práctica de las } \\
\text { propuestas de mejora por parte de } \\
\text { los equipos; } \\
\text { b) éxitos tempranos; } \\
\text { c) vincular las técnicas y las herramientas } \\
\text { con las metas; } \\
\text { d) gestionar el cambio cultural y de } \\
\text { valores; es decir, la implementación } \\
\text { del Kaizen en todos los aspectos de } \\
\text { la organización }\end{array}$ & $\begin{array}{l}\text { - Equipos de mejora } \\
\text { - Estandarización } \\
\text { - Control Total de Calidad } \\
\text { - Mantenimiento Productivo } \\
\text { Total } \\
\text { - Justo a Tiempo (JIT) }\end{array}$ \\
\hline
\end{tabular}


grupal, y, 3) el Kaizen individual. Asimismo, Imai (1986, 1997) proporciona una serie de conceptos o características con el fin de implementar el Kaizen: a) el Kaizen y la Gerencia, donde la responsabilidad de innovar y mejorar queda en la alta gerencia, mientras que el mantenimiento de los estándares de trabajo con sus respectivas mejoras incrementales, está en los mandos intermedios y los trabajadores; b) el enfoque en los procesos versus los resultados; c) seguir continuamente los ciclos de mejora PDCA (acrónimo de plan, do, check, act) y de estandarización SDCA (acrónimo de standardize, do, check, act) por parte de todos los actores de una organización; d) primero la calidad; e) hablar con datos (control estadístico) y f) el proceso siguiente es el cliente.

Un resumen de las investigaciones analizadas que siguen la vertiente japonesa se presenta en la Tabla 1.

\subsubsection{El Kaizen en su orientación occidental}

Aunque la literatura sobre el Kaizen desde su orientación occidental (como término de Mejora Continua -MC-) es más abundante que la de su orientación japonesa, sigue siendo señalada por algunos autores (Savolainen 1999; Bhuiyan y Baghel 2005), con una fuerte necesidad de ampliar los fundamentos teóricos de la misma. La razón de esta afirmación, es porque el Kaizen, entendido como Mejora Continua, tiende a ser utilizado en su concepción, como un elemento general, atributo o factor crítico de otras aproximaciones gerenciales tales como la Gestión por Calidad Total (TQM), el Pensamiento Esbelto (Lean Thinking). Sin embargo, los trabajos de Lillrank y Kano (1989), Schroeder y Robinson (1991), Bessant y Caffyn (1997), Lindberg y Berger (1997), Caffyn (1999), Savolainen (1999), Kaye y Anderson (1999), Bessant (2003), Jorgensen et al. (2003) y Jager et al. (2004) han representado importantes contribuciones que han ayudado a reducir dicha brecha teórica.

De esta manera, un aporte importante a esta orientación del Kaizen desde sus orígenes, se encuentra en dos libros fundamentales, el de Vogel de 1979 denominado Japón como el número uno (Japan as Number One por su nombre en inglés), y el libro de Pascale y Athos de 1981 denominado El arte de la gestión japonesa (The Art of Japanese Management por su nombre en inglés), lo cual incrementó en gran medida la orientación de occidente por el concepto japonés de la mejora (Styrhe 2001: 797). Asimismo, otro trabajo relevante fue el de Schroeder y Robinson (1991), los cuales explican un posible origen del concepto de 
Mejora Continua en las iniciativas del gobierno de los Estados Unidos de América en los años cuarenta y cincuenta por potenciar la productividad industrial a nivel país. Por último, Lindberg y Berger (1997) por su parte, estudiaron la aplicabilidad de las iniciativas de Mejora Continua en varios tipos de organizaciones suecas. Estos autores encontraron que las organizaciones suecas van más allá de crear una estructura "paralela" o "virtual" a la existente durante las actividades de Kaizen, como lo hacen las organizaciones que utilizan el enfoque japonés del término, ya que dichas actividades, como por ejemplo, la aplicación de los equipos de mejora, son integradas en el trabajo cotidiano.

En una línea similar, el modelo de "High Involvement Innovation (HII)" desarrollado por el equipo del proyecto "Continuous Improvement Research for Competitive Advantage (CIRCA)", entre 1992 y 1997 en la Universidad de Brighton en el Reino Unido, provee una poderosa guía para evaluar la utilidad de la implementación de la Mejora Continua (Caffyn 1999). Los trabajos de Bessant y Caffyn (1997) que se concentran en el libro del primer autor (Bessant 2003), proporcionan un modelo en el que la Mejora Continua o el Kaizen bajo el enfoque occidental, no es una realidad estática, sino que evoluciona de manera dinámica a través de cinco niveles (nivel previo a la $M C$, nivel de estructura, nivel de enfoque en la meta, nivel proactivo, y nivel de capacidad organizacional de la $M C$ ), y en cada uno de ellos, se encuentran comportamientos específicos que aparecen al momento de implementar con éxito la Mejora Continua. Para estos autores cada uno de estos comportamientos, que son desarrollados a lo largo del tiempo por la organización, representan elementos necesarios para desarrollar a la Mejora Continua como una capacidad de la organización. Al respecto, Caffyn (1999: 1142) define la capacidad de la Mejora Continua como "la habilidad que tiene la organización para obtener ventaja competitiva a través de extender la innovación en una proporción significativa de sus miembros". Otros autores, la han comenzado a visualizar de igual manera como una "Capacidad Dinámica" de la organización (Bessant and Francis 1999; Suárez-Barraza et al. 2008).

Dentro de esta orientación más occidental, el Kaizen también ha sido abordado desde un ángulo gerencial y organizacional más practico, delimitando al término mismo, en forma de metodología y/o técnica conformada por conjunto de herramientas necesarias para eliminar las actividades que no agregan valor a los procesos de trabajo, los llamados "mudas" en japonés. Entre ellas están el Kaizen Blitz (Tillinghurst 1997; 
Tabla 2. Aproximaciones prácticas del Kaizen en su vertiente occidental

\begin{tabular}{|c|c|c|c|}
\hline $\begin{array}{l}\text { Aproximación } \\
\text { práctica }\end{array}$ & Autores & $\begin{array}{l}\text { Características y elementos de } \\
\text { la aproximación práctica }\end{array}$ & $\begin{array}{c}\text { Técnicas y } \\
\text { herramientas del } \\
\text { Kaizen utilizadas }\end{array}$ \\
\hline Kaizen Blitz & \begin{tabular}{|l} 
- Laraia et al. \\
(1999) \\
- Cuscela \\
(1998) \\
- Sheridan \\
(1997) \\
- Tillinghurst \\
(1997)
\end{tabular} & $\begin{array}{l}\text { Esta metodología del Kaizen en su orientación occidental, } \\
\text { consiste en una serie de eventos que se realizan durante } \\
\text { un grupo relativamente pequeño de días (entre } 2 \text { y 5), } \\
\text { con el objeto de encontrar y mejorar cualquier muda } \\
\text { que se logre identificar en los procesos de trabajo del } \\
\text { shopfloor. Asimismo, el Kaizen Blitz requiere de cinco } \\
\text { elementos centrales para su aplicación: 1) utilizar una } \\
\text { perspectiva estratégica para aplicar el Kaizen Blitz (crear } \\
\text { un plan o programa específico); } 2 \text { ) aplicar los eventos } \\
\text { Kaizen para transformar la cultura de la organización, } \\
\text { a través del facultamiento (empowerment) de los } \\
\text { empleados; } 3 \text { ) asegurarse que todo mundo entiende } \\
\text { los principios y técnicas del Kaizen Blitz al momento } \\
\text { de aplicarse; 4) reclutar las personas adecuadas para } \\
\text { la Oficina de Promoción del Kaizen (KPO); y finalmente, } \\
\text { 5) buscar la manera de eliminar las resistencias al cambio } \\
\text { por parte de los empleados, incluyendo el miedo ante } \\
\text { lo desconocido y las presiones por reducir los costes }\end{array}$ & $\begin{array}{l} \\
\text { - Equipos de Mejora } \\
\text { - Rediseño de procesos } \\
\text { (los blitzes) } \\
\text { - Planes de Acción de } \\
\text { Mejora } \\
\text { - Value Stream Mapping } \\
\text { - Técnicas y herramientas } \\
\text { estadísticas } \\
\text { - Nivelado de flujos de } \\
\text { procesos } \\
\text { - } 7 \text { herramientas básicas } \\
\text { de calidad }\end{array}$ \\
\hline $\begin{array}{l}\text { Gemba-Kaizen } \\
\text { workshops }\end{array}$ & $\begin{array}{l}\text { - Wennecke } \\
(2008) \\
- \text { Imai (2007) } \\
\text { - Lewis (2007) } \\
\text { - Ortíz (2006) } \\
\text { - Bodek (2002) } \\
\text { - Montabon } \\
\text { (1995) } \\
\text { - Melnyck et } \\
\text { al. }(1998)\end{array}$ & $\begin{array}{l}\text { Los Gemba-Kaizen workshops representan un verdadero } \\
\text { vínculo entre la estrategia de la organización y la } \\
\text { operación cotidiana, con un objetivo central en mente: } \\
\text { la eliminación del muda en corto tiempo. Los elementos } \\
\text { claves son: a) conformar un equipo de mejora; b) } \\
\text { planificar y anticipar los resultados; c) vínculo } \\
\text { estratégico; d) participación de los empleados y e) } \\
\text { nombrar un líder del evento }\end{array}$ & $\begin{array}{l}\text { - Equipos de Mejora } \\
\text { - Rediseño de procesos } \\
\text { (los blitzes) } \\
\text { - Planes de Acción de } \\
\text { Mejora } \\
\text { - Value Stream Mapping } \\
\text { - Técnicas y herramientas } \\
\text { estadísticas } \\
\text { - Nivelado de flujos de } \\
\text { procesos }\end{array}$ \\
\hline Office Kaizen & - Lareu (2003) & $\begin{array}{l}\text { Se sustenta en un modelo que el autor denomina: } \\
\text { SLIM-IT. Término que es entendido como la aplicación } \\
\text { de diferentes mecanismos que define el autor como } \\
\text { "Structure, Lean daily management system, Metrics, } \\
\text { Mentoring, Tools, Training, Team-work, and Information } \\
\text { Technology". El objetivo de esta metodología del } \\
\text { Kaufman Consulting Group es la reducción de los } \\
\text { costes operativos y de gestión de las organizaciones, } \\
\text { mediante la eliminación de los mudas, al igual que las } \\
\text { anteriores }\end{array}$ & $\begin{array}{l}\text { - Equipos de Mejora } \\
\text { - Rediseño de procesos } \\
\text { (los blitzes) } \\
\text { - Planes de Acción de } \\
\text { Mejora } \\
\text { - Value Stream Mapping } \\
\text { - Técnicas y herramientas } \\
\text { estadísticas } \\
\text { - Aplicación de } \\
\text { Tecnologías de } \\
\text { Información } \\
\text { - Tutoreo (coaching) }\end{array}$ \\
\hline Kaizen Teian & \begin{tabular}{|l} 
\\
\\
- Japan Human \\
Relations \\
Association \\
$(1990)$
\end{tabular} & $\begin{array}{l}\text { Dicha aproximación, va mucho más allá del simple } \\
\text { sistema de sugerencias de empleados surgido en el } \\
\text { ejército norteamericano en los años cuarenta y cincuenta. } \\
\text { De hecho, el Kaizen Teian opera desde un ángulo más } \\
\text { integral a la gestión de la organización bajo tres } \\
\text { principios básicos: 1) un sistema de participación, donde } \\
\text { los empleados participan de manera voluntaria en la } \\
\text { mejora de su trabajo; } 2 \text { ) desarrollo de habilidades, donde } \\
\text { la gerencia tiene como responsabilidad entrenar a los } \\
\text { empleados en todo momento y los empleados de } \\
\text { aprender a través de la práctica. Y finalmente, 3) creación } \\
\text { de la fuerza impulsora, compuesto por las políticas de } \\
\text { la alta dirección, participación de directivos, el desarrollo } \\
\text { de objetivos y de mecanismos de recompensas } \\
\text { (preferentemente no monetarios) }\end{array}$ & \begin{tabular}{|l} 
\\
- Sistema de Propuesta \\
- Equipos de Mejora \\
- Entrenamiento activo \\
- Sistema de recompensas \\
- Compromiso de la alta \\
dirección
\end{tabular} \\
\hline
\end{tabular}


Cuscela 1998); los Gemba-Kaizen workshops or weeks, o también conocidos como "short-cycle Kaizen, rapid Kaizen or Kaikaku» (Montabon 1995; Sheridan 1997; Melnyck et al. 1998; Imai 2007); el Office Kaizen (Lareu 2003); y finalmente, el Kaizen Teian (Japan Human Relations Association 1990). En la Tabla 2 se describen las características de cada uno de ellos.

\section{TRES DIFERENTES ESFERAS CONCÉNTRICAS DEL KAIZEN}

Como resultado del análisis del Kaizen en la literatura existente, y siguiendo el esquema propuesto por Dean y Bowen (1994) se proponen tres diferentes esferas concéntricas (para realizar una analogía) por las que puede ser comprendido el término de Kaizen: 1) el Kaizen como «filosofía gerencial», 2) el Kaizen como un elemento del TQM, y 3) el Kaizen como principio teórico de metodologías y técnicas de mejora.

gerencial»

\subsection{Esfera concéntrica del tipo 1: el Kaizen como «filosofía}

La primera de ellas, percibe al Kaizen como «filosofía gerencial», que involucra una serie de principios y valores que soportan al sistema de gestión de la organización. En otras palabras, dentro de esta sombrilla se encuentran todas las prácticas, técnicas o herramientas de gestión, consideradas en su origen como japonesas, dentro de las cuales se incluyen la Gestión por Calidad Total de toda la Empresa (CWQC) (Imai 1989: 40) (ver Figura 1). De hecho, algunos autores indican que el Kaizen como filosofía de gestión ha sido el resultado del desarrollo de las ideas del control de calidad de las empresas de manufactura en los años sesenta y setenta en el Japón, donde los trabajos de Ishikawa, Mizuno, Shingo, Miyauchi y Ohno fueron fundamentales (Imai 1986; Sawada 1995). $Y$ tal como se observa en los trabajos empíricos encontrados en la literatura, parece ser, que esta perspectiva del Kaizen es la que sigue vigente en las organizaciones japonesas de alto desempeño (Cheser 1998; Brunet 2000; Brunet y New 2003; Liker 2004; Vonk 2005; Hino 2006). Algunos autores en esa misma línea, han indicado que el Kaizen entendido como «filosofía gerencial», representa los cimientos y la base de los valores y la cultura que se vive en el día a día de una las principales de estas empresas, Toyota Motor Company, a través de su modelo de gestión denominado: «Toyota Way 2001» (Hino 2006: 82). 
De acuerdo al análisis de la literatura, el Kaizen como «filosofía gerencial» se caracteriza por entender la gestión de una organización como el mantenimiento y la mejora de los estándares de trabajo. Para Imai (1986: 74), no puede existir el Kaizen sin la estandarización, es decir, las mejoras incrementales y acumulables sólo se consiguen cuando los estándares se encuentran establecidos y sostenidos mediante el trabajo cotidiano. Una indicación importante de esta visión pragmática del trabajo, bajo esta esfera del Kaizen, es que los estándares, deben ser seguidos por cada uno de los empleados y por lo tanto, el trabajo de la gerencia se centra en su seguimiento. Desde este ángulo, es como se entiende la disciplina en el trabajo (Imai 1986: 75). De esta manera, la estandarización, la comunicación inter-funcional, el orden, la disciplina y la eliminación de los mudas representan los requisitos básicos de operación del Kaizen bajo esta sombrilla (Imai 1997; Brunet y New 2003; Aoki 2008).

Otras de las características esenciales de esta esfera concéntrica del Kaizen, es que el mantenimiento y la mejora de los estándares se realiza a través del involucramiento y participación de "todos" los empleados de la organización. El medio que utilizan las organizaciones bajo esta perspectiva son los equipos de mejora (el Kaizen orientado al grupo), y el sistema de propuestas (el Kaizen orientado al individuo). Esta característica se presenta y así se sugiere en la literatura, con una motivación predominante intrínseca (Berger 1997), sustentada en el voluntarismo o Jishusei (palabra japonesa) de los empleados (Lillrank y Kano 1989). La fuerza impulsora de esta motivación de acuerdo a la literatura, es el concepto de de «zero defect», en el cual los trabajadores de manera espontánea y automática buscan mejorar los procesos de trabajo (Brunet y New 2003).

Finalmente, las mejoras se realizan sobre los procesos de trabajo directamente en el área de trabajo, es decir, en el lugar de trabajo (en el "gemba" en su término japonés), a través del trabajo disciplinado y constante de los empleados. Las habilidades de observación para encontrar problemas y mudas, y la oportunidad de experimentar las mejoras en ambientes controlados se presentan en todo momento. Para ello, se hacen uso de diferentes técnicas que tratan de conseguir el mantenimiento y la mejora de los estándares, donde cada una de ellas, se encuentran estrechamente vinculadas a los principios rectores del Kaizen (ver Figura 1). El soporte de toda esta primera perspectiva se encuentra en el claro vínculo entre la alta dirección y los empleados con respecto a las 
actividades del Kaizen como «filosofía gerencial»; el medio para realizar este vinculo es el despliegue de políticas y objetivos desde la alta dirección hasta el último empleado del área de trabajo, conocido como: "Hoshin Kanri". La Figura 1 resume lo anteriormente descrito.

Figura 1. Esfera concéntrica del tipo 1: el Kaizen como «filosofía gerencial»

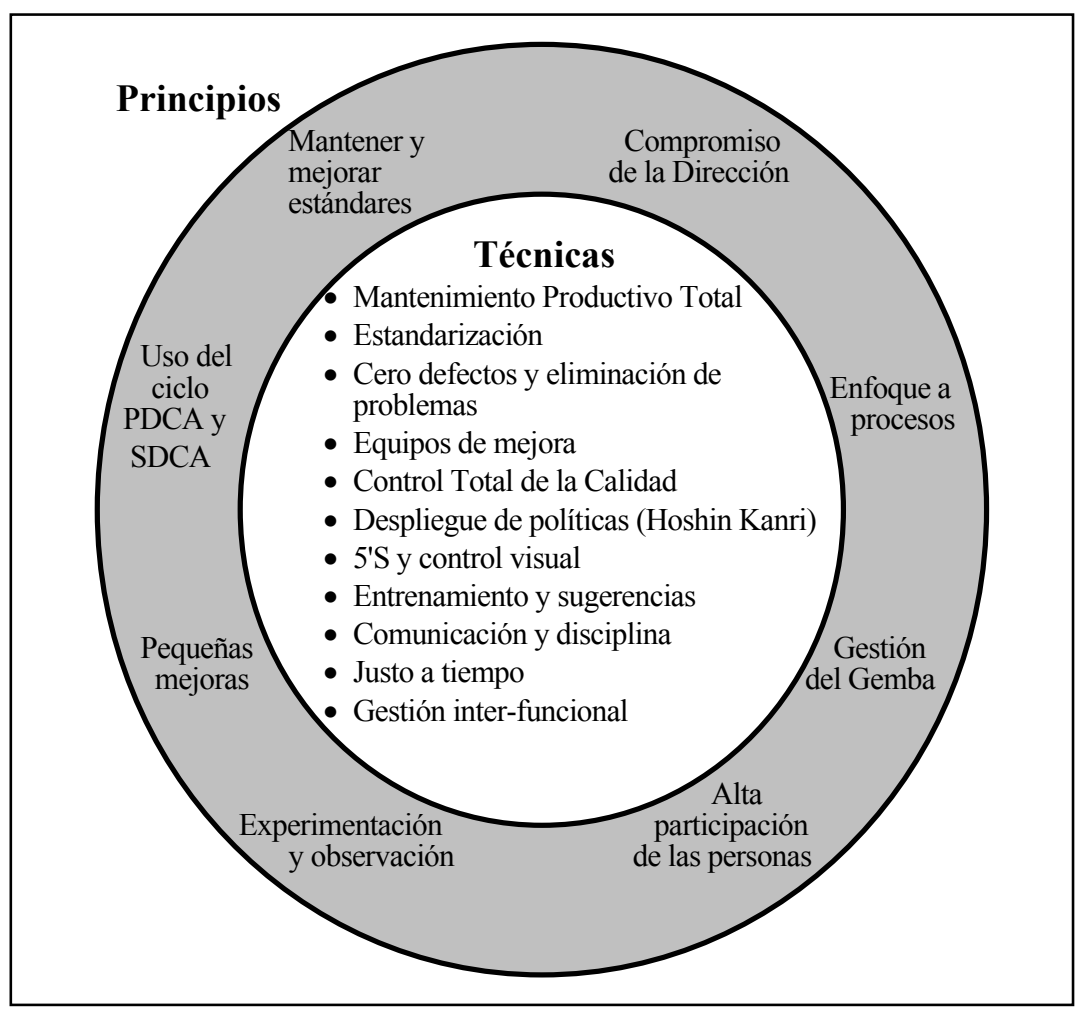

3.2. Esfera concéntrica del tipo 2: el Kaizen como un elemento del TQM

La segunda esfera concéntrica identificada, delimita al Kaizen como un elemento más de la Gestión por Calidad Total (TQM). La literatura indica que las raíces de los programas de mejora en occidente se pueden trazar hasta los trabajos del gobierno norteamericano en el llamado "Training Within Industry" durante la Segunda Guerra Mundial; este programa incluyó el entrenamiento y educación de los supervisores con técnicas de control estadístico y mejora continua de los procesos (Schoeder y Robinson 1991). Más tarde, dicho programa fue introducido en el Japón en los años cincuenta, por varios autores reconocidos en el tema, como Deming, 
Juran y otros (Ishikawa 1986; Robinson 1990). Para el propio Deming (1986: 49), esta esfera (la Mejora Continua dentro del TQM) era entendida dentro sus 14 principios rectores como "mejorar constantemente el sistema de producción y de servicios". Mientras que para Juran (1990) dicha esfera, sólo formaba parte de su "Trilogía para la Calidad", entendida como la Mejora de la Calidad o la ruptura de los procesos, después de que han existido dos fases anteriores de planificación y control de los mismos.

El debate sobre los fundamentos teóricos y de los elementos del TQM continúa hasta la fecha, a pesar de los trabajos empíricos que han aportado cierta luz a la construcción de la teoría de esta perspectiva gerencial (del TQM). Varios de estos trabajos han contribuido a determinar más concretamente que la Mejora Continua es un elemento del TQM, lo cual, aparece reflejado en algunos constructos con correlaciones significativas (Flynn et al. 1994; Powell 1995; Ahire et al. 1996; Quazi et al. 1998). En este sentido, Dean y Bowen (1994: 394) indican que uno de los tres elementos que conforman el TQM es la Mejora Continua (Kaizen), además del enfoque en el cliente y el trabajo en equipo. Para este autor, la Mejora Continua es entendida como un elemento del TQM, lo que significa un compromiso constante de la organización por examinar sus procesos técnicos y administrativos, con el fin de buscar mejores métodos de trabajo. Para alcanzar este objetivo, múltiples técnicas, incluyendo el control estadístico de procesos, la Historia de la Calidad (metodología para solucionar problemas -QC Story por su término en inglés-), y los diagramas de flujo, se asocian con este elemento del TQM (ver Figura 2). Otros autores han señalado que la Mejora Continua (el Kaizen) también es un elemento o un "valor" fundamental del TQM, cuando este último se comporta como un sistema de gestión (Hakes 1991; Ho and Fung 1994; Hellsten and Klefsjö 2000). Otras investigaciones realizadas indican que la Mejora Continua puede ser vista como una capacidad organizacional, ya sea formando parte del TQM o como otro tipo de programa de mejora o innovación (Bessant y Caffyn 1997; Bessant 2003). Desde dichos estudios, el Kaizen entendido como Mejora Continua, no se percibe como simplemente un proceso lineal, en el que una vez que se ha implantado un esfuerzo de mejora, este debe mantenerse y mejorarse de manera continua. Por el contrario, se entiende como un proceso dinámico evolutivo, compuesto por una serie de niveles por los que una organización puede transitar al aplicar la Mejora Continua como una capacidad de la organización. Bien conducido, este proceso dinámico puede llevar a la organización a alcanzar una capacidad dinámica de la organización que le produzca una 
ventaja competitiva (Bessant y Francis 1999; Suárez-Barraza et al. 2008). En la Figura 2 se concentra lo anteriormente descrito.

Figura 2. Esfera concéntrica del tipo 2: el Kaizen como un elemento del TQM

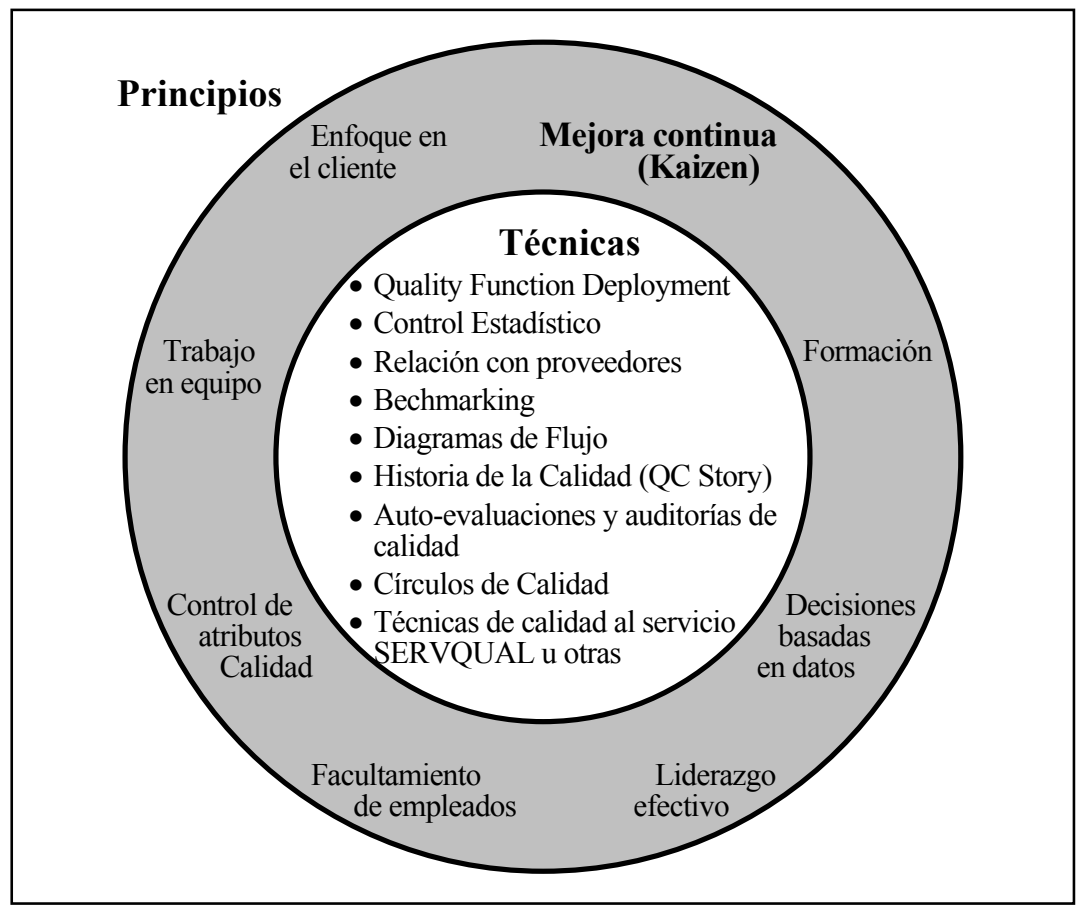

3.3. Esfera concéntrica del tipo 3: el Kaizen como principio teórico de metodologías y técnicas de mejora

La tercera y última esfera concéntrica que afloró del análisis de la literatura, utiliza al Kaizen como sustento teórico para aplicar metodologías y/o técnicas que tienen como propósito básico el eliminar el desperdicio (Mudas). Por ello, a través de la eliminación del muda se busca mejorar la calidad de los procesos y los productos, reducir el lead time, optimizar la entrega a tiempo de los productos e incluso, mejorar el flujo de dinero. De acuerdo a la literatura analizada se lograron identificar al menos cuatro metodologías y/o técnicas que están bajo esta sombrilla: el Kaizen Blitz, los gemba-Kaizen, el Office Kaizen y el Kaizen Teian.

Las características principales de esta tercera perspectiva son en primer lugar, el enfoque al corto plazo del mismo. Se trata por 
ende, de eliminar mudas en workshops o eventos Kaizen intensivos (sólo algunos días), en los cuales se utilizan las ideas de mejora de los empleados que conocen los procesos de trabajo a fondo (Sheridan 1997; Cuscela 1998). De esta manera, el conseguir eliminar los mudas en tiempos bastante cortos permite al mismo tiempo, la participación de los empleados y la obtención de resultados positivos de manera rápida (quick wins), lo que representa una segunda característica. La tercera característica es que el enfoque que se busca es la eliminación de mudas de manera transversal, a lo largo del proceso (cross functional approach), por lo que además, cada proyecto de mejora es liderado por algún empleado que es reconocido por su capacidad técnica, generalmente empleados veteranos y con amplia experiencia. Por último, para el seguimiento de los proyectos de mejora, es decir, después de los cuatro días se conforman una serie de comités o áreas staff que monitorean lo previamente implantado (ver Figura 3).

Figura 3. Esfera concéntrica del tipo 3: el Kaizen como principio teórico de metodologías y técnicas de mejora

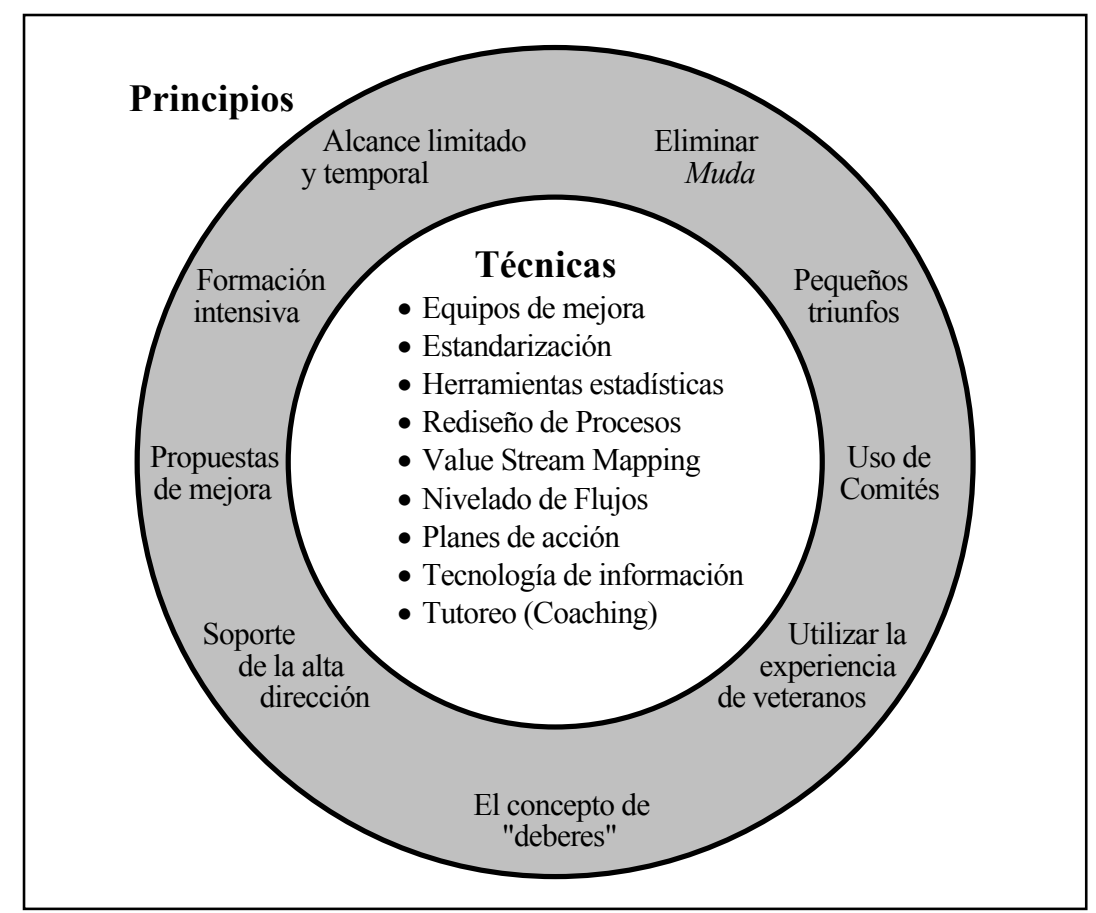

De acuerdo a lo analizado en la literatura, esta perspectiva del Kaizen bajo todas estas metodologías y técnicas, toma los principios 
más operativos del Kaizen como «filosofía gerencial» (primera sombrilla, ver Figura 1), y los lleva a la práctica mediante las técnicas y herramientas que la sombrilla o perspectiva 1 tiene, además de otras. Por esta razón, el alcance y enfoque desde un punto de vista organizacional es micro, centrado en la operación y los procesos de trabajo, y en la participación de los empleados. Por otro lado, es importante señalar que el Kaizen Office y el Kaizen Teian tienden en su alcance a un enfoque más organizacional que abarca otros elementos organizacionales tales como la estructura organizacional, la medición del rendimiento de toda la organización y algunos aspectos de la estrategia (Japan Human Relations Association 1990; Lareu 2003). Aún así, se centran en los aspectos operativos del Kaizen como «filosofía gerencial». Esta última esfera, se esquematiza en la Figura 3.

\section{CONCLUSIONES Y TEMAS PARA FUTURAS INVESTIGACIONES}

Las tres esferas concéntricas propuestas en este artículo representan una primera aproximación por intentar entender al Kaizen, por lo que es evidente, que esta forma de agrupación realizada a través de un análisis teórico puede tomar diferentes formas de plantearlas dependiendo del ángulo con que se aborde. En este sentido, somos consientes que es muy difícil realizar una clara separación teórica y analítica entre cada una de las esferas concéntricas, ya que algunos principios y técnicas se presentan en una o en otra esfera (ver Figura 4). Este posible solapamiento es el resultado de cómo el Kaizen ha ido evolucionando a lo largo de los años tanto en la arena teórica como en el campo práctico. De hecho, también puede ser el resultado del pobre entendimiento que ha tenido en occidente esta aproximación japonesa. No obstante, esperamos que con nuestra propuesta se comience a generar un debate productivo acerca de este tema poco explorado en la literatura académica, señalando que este primer intento de tipología ayude a futuras investigaciones del Kaizen. 
Figura 4. Análisis cruzado de las tres esferas concéntricas del Kaizen

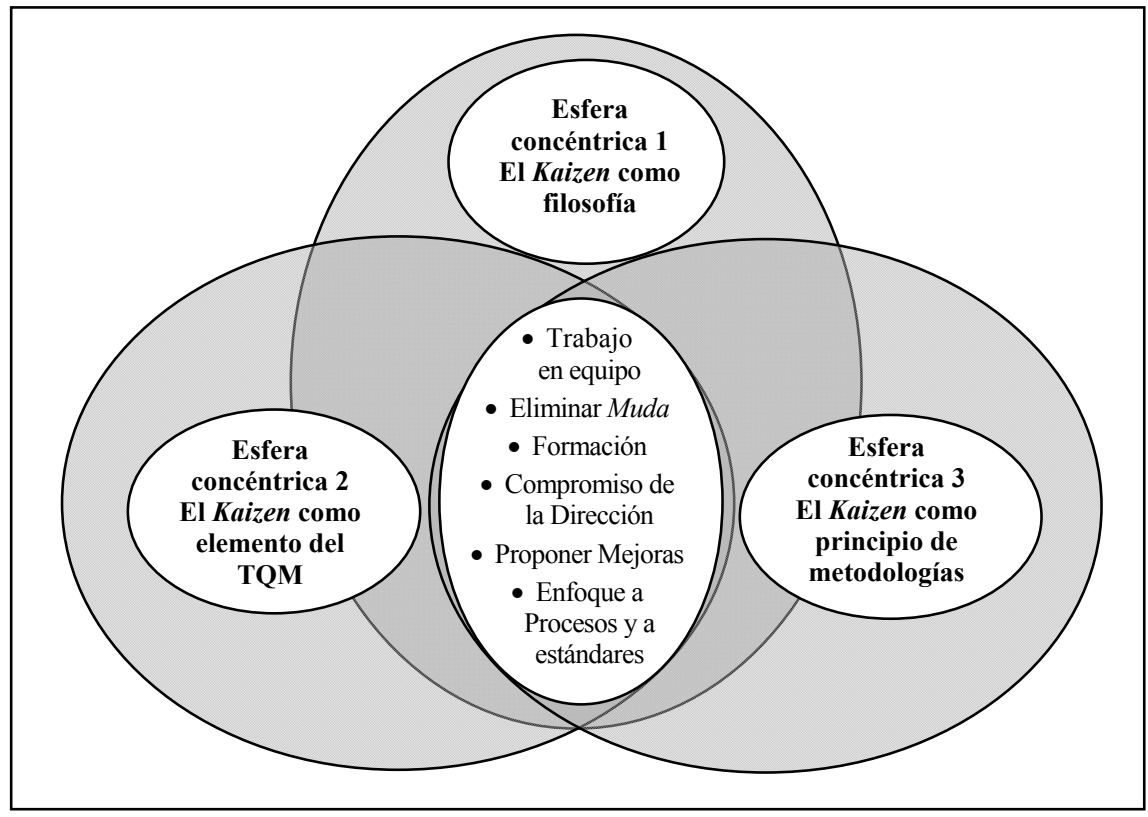

De esta manera, cerramos este artículo con una serie de posibles temas o aspectos que han surgido de esta investigación, y que merecen por tanto atención, estableciendo con ello las bases para futuras investigaciones teóricas y/o prácticas.

\subsection{Clarificación del concepto del Kaizen}

De acuerdo a los resultados de Brunet y New (2003) parece que el Kaizen sigue siendo un concepto poco uniforme en su comprensión, incluso para las organizaciones japonesas. Quizás porque sus principios básicos, descritos por Imai $(1989,1997)$ y formulados por Berger (1997) como tal, son adaptados por los gerentes de las organizaciones de acuerdo a las necesidades de cada organización (Brunet y New 2003). Esto ha generado cierta confusión al momento de entenderlo, y quizás pueda explicar los fallos al momento de exportarlo a un entorno de organizaciones occidentales (Spear 2004). Lillrank (1995) indica al respecto, que la transferencia de las prácticas organizacionales japonesas, entre las que se puede situar al Kaizen, requiere de una profunda clarificación y reinterpretación del concepto antes de llevarla a la práctica. Y a pesar de las últimas investigaciones que la literatura muestra por explicar y perfilar al Kaizen 
(Berger 1997; Brunet 2000; Brunet y New 2003; Imai 2007), se sigue haciendo necesario y requerido un esfuerzo más detallado y profundo por clarificarlo y delimitarlo. Finalmente, vale la pena resaltar que la clarificación del concepto del Kaizen en futuras investigaciones, no es cuestión de tan sólo semántica teórica. Por el contrario, resulta fundamental para las organizaciones entender de qué estamos hablando con el fin de evitar errores, frustraciones o decepciones al momento de llevarlo a la práctica.

\subsection{Aplicación del Kaizen en las organizaciones}

Diferentes metodologías y técnicas se identificaron de la literatura, para aplicar el Kaizen en las organizaciones, desde el Kaizen Blitz, los talleres Gemba Kaizen, el Kaizen Office, hasta aquellos basados en un sistema de propuestas de los empleados, como el Kaizen Teian, o como parte de aproximaciones más integrales como el TQM. $Y$ aunque cada una de ellas muestra a su manera, la forma detallada de aplicarlo, algunos principios identificados en la esfera concéntrica del Kaizen como "filosofía gerencial" (ver Figura 1) se quedan ausentes al momento de implementar dichas metodologías. En este sentido, las organizaciones de hoy lo que buscan son principios rectores y guías desde una visión más integral y holística, que tan sólo "recetas o metodologías preestablecidas". Por ello, la idea de un continuo esfuerzo por mejorar en el día a día para hacer frente a los cambios y presiones del entorno externo (Wittenberg 1994; Imai 2007), cobra mayor fuerza desde una óptica en el que el Kaizen, se puede aplicar como una serie de principios rectores que guíe la mejora y el aprendizaje, sobre todo de aquellos que se presentan en las tres esferas, los cuales se pueden considerar sus "piedras angulares" (ver Figura 4).

Asimismo, debido a la diversidad mostrada de metodologías relacionadas con el Kaizen que se han aplicado y que se aplican en las organizaciones, se hace necesario el continuar en la búsqueda de los principios rectores del Kaizen, para conseguir aplicaciones e incluso transferencias más efectivas en las organizaciones dentro y fuera del Japón, tal como concluye Aoki (2008). Finalmente, se hace necesario realizar un trabajo más profundo por identificar los factores críticos de éxito del Kaizen, lo que permitirá comprenderlo y perfilarlo aún mejor. Así ha sucedido con la construcción de teoría para conceptos como el TQM (Flynn et al. 1994; Powell 1995; Ahire et al. 1996; Quazi et al. 1998). Además, el trabajo en la identificación de los factores críticos tendrá implicaciones 
prácticas para aquellas organizaciones que estén pensando implementar o que hayan aplicado cualquiera de las tres esferas del Kaizen.

\subsection{Pluralidad de organizaciones al momento de aplicarlo}

De acuerdo a la exploración de la literatura realizada, se observó que prácticamente sólo las grandes corporaciones, multinacionales y algunos de sus proveedores tanto japoneses como occidentales son en las que se reportan estudios acerca de la aplicación del Kaizen (Tanner y Roncarti 1994; Malloch 1997; Cheser 1998; Brunet y New 2003; Lewis 2007; Wennecke 2008). Por este motivo existe una gran área de oportunidad de estudio que requiere ser analizado como lo son las pequeñas y medianas empresas. En una de sus últimas conferencias el profesor Imai (2007) aportó al respecto, un caso de una empresa de tamaño medio en la India, en dónde el Kaizen se había desarrollado con relativo éxito. Por otra parte, también se hace necesario estudiar dentro de las grandes corporaciones cómo se aplican el Kaizen en sus diferentes áreas, departamentos o unidades y procesos, con el fin de observar el impacto específico, así como, el posible despliegue de la aplicación en toda la organización, si es que se presenta, y si no, comprender, por qué no se presenta.

Por último, Montabon (2005) también ha explorado en las áreas de servicio la aplicación del Kaizen de la tercera perspectiva del Kaizen, lo que comienza a enriquecer las investigaciones haciendo esta distinción entre manufactura y servicio. En esa misma línea, también se puede abrir una línea de investigación acerca de la investigación del Kaizen en el sector público, que algunos autores han comenzado a explorar (Suárez-Barraza y Ramis-Pujol 2008; Imai 2007).

\section{BIBLIOGRAFÍA}

AHIRE, S. \& D. GHOLAR (1996) "Development and Validation of TQM Implementation Constructs", Decision Sciences, 27, No. 1, pp. 23-56.

AOKI, K. (2008) "Transferring Japanese Kaizen activities to overseas plants in China", International Journal of Operation \& Production Management, 28, No. 6, pp. 518-539. 
BATEMAN, N. (2005) "Sustainability: the Elusive Element of Process Improvement", International Journal of Operations \& Production Management, 25, No. 3, pp. 261-276.

BeRger, A. (1997) "Continuous Improvement and Kaizen: Standardizations and Organizational Designs", Integrated Manufacturing System, 8, No. 2, pp. 110-117.

BESSANT, J. (2003) High Involvement Innovation. Chichester West Sucess England: John Wiley and Songs Ltd.

- and S. CAFFYN (1997) "High-Involvement Innovation Through Continuous Improvement", International Journal Technology Management, 14, No. 1, pp. 7-28.

- and D. FRANCIS (1999) "Developing strategic continuous improvement capability", International Journal of Operations \& Production Management, 19, No. 11, pp. 1106-1119.

BHUIYAN, N. and A. BAGHEL (2005) "An Overview of Continuous Improvement: From the Past to the Present", Management Decision, 43, No. 5, pp. 761-771.

BodeK, N. (2002) "Kaizen: Kazam!", $T+D$, 56, No. 1, pp. 60-61.

BRUNET, A.P. (2000) "Kaizen: From Understanding to Action", Ponencia realizada en el Institution of Electrical Engineers, London., UK, IEE Savoy Place.

- and S. New (2003) "Kaizen in Japan: an Empirical Study", International journal of Operations \& Production Management, 23, No. 12, pp. 14261446.

CAFFYN, S. (1999) "Development of a Continuous Improvement Selfassessment Tool", International Journal of Operations \& Production Management, 19, No. 11, pp. 1138-1153.

CHESER, R. (1994) "Kaizen is More Than Continuous Improvement", Quality Progress, 27, pp. 23-25.

- (1998) "The Effect of Japanese Kaizen on Employee Motivation in US Manufacturing", The International Journal of Organizational Analysis, 6, No. 3, pp. 197-217.

COX, J. and J.H. BLACKSTONE (2002) APICS On line dictionary [http://members.apics.org/Publications/dictionary/articlesearch.asp].

CROSBY, P. (1979) Quality is Free. New York: McGraw-Hill.

CUSCELA, K. (1998) "Kaizen Blitz: Attacks Work Processes at Dana Corp", IIEE Solutions, 30, No. 4, pp. 29-31. 
DaHLGaARD, J.J. and S.M. DAHLGAARd-PARK (2006) "Lean Production, Six Sigma Quality, TQM and Company Culture". The TQM Magazine, 18, No. 3, pp. 263-281.

DEAN, J.W.J. and D.E. BOWEN (1994) "Management theory and total quality improvement research and practice through theory development", Academy of Management Review, 19, No. 3, pp. 392-418.

DEMING, W.E. (1986) Out of the crisis. Cambridge, Massachusetts: MIT/CAES.

ELGAR, T. and C. SMITH (1994) Global Japanization: The Transnational Transformation for the Labour Process. London: Routledge.

FARLEY, C. (1999) "Despliegue de Políticas del KAIZEN". XI Congreso de Calidad Total organizado por el Centro de Productividad de Monterrey, Monterrey Nuevo León México. Fundación Mexicana de la Calidad Total y Centro de Productividad de Monterrey.

FeIGEMBaUm, A.C. (1991) Total Quality Control. New York: McGraw-Hill.

FLYNN, B.B.; S. SAKAKIBARA and R.G. SCHROEDER (1994) "A Framework for Quality Management Research and an Associated Measurement Instrument", Journal of Operations Management, 11, No. 4, pp. 339366.

GARVIN, D.A. (1987) "Competing on the Eight Dimensions of Quality", Harvard Business Review, 65, No. 1, pp. 101-109.

GondHAleKar, S.; S. BABU and N.B. GodReJ (1995) "Towards using Kaizen Process Dynamics: a Case Study", International journal of Quality \& Reliability Management, 12, No. 9, pp. 192-209.

HAKES, C. (1991) Total Quality Management: A Key to Business Improvement. London: Chapman \& Hall.

HeLlSTen, U. and B. KLEFSJÖ (2000) "TQM as a Management System Consisting of Values, Techniques and Tools", The TQM Magazine, 12, No. 4, pp. 238-244.

HINO, S. (2006) Inside the Mind of Toyota. New York: Productivity Press.

Ho, S. and C. FUnG (1994) "Developing a TQM Excellence Model", The TQM Magazine, 6, No. 6, pp. 24-30.

- and S. CITMILL (1996) "Japanese 5-S practice", The TQM Magazine, 8, No.1, pp. 45-53.

IMAI, M. (1986) Kaizen-The key to Japan's Competitive Success. New York: Random House.

- (1989) Kaizen, la clave de la ventaja competitiva japonesa. México, D.F.: CECSA. 
- (1997) Gemba Kaizen. New York, NY: Mcgraw-Hill.

- (2006) ¿What is Total Flow Management under Kaizen Approach? 3rd. Day of Kaizen Course. Barcelona, Spain: Kaizen Institute Spain.

- (2007) "Mejorar la calidad es la mejor forma de reducir los costes", Diario de Negocios la Gaceta, 13 de diciembre, p. 36.

ISHIKAWA, K. (1986) What is Total Quality Control? The Japanese Way. Englewood Cliffs, NJ: Prentice Hall.

Jager, B. de; C. Minnie, J. Jager, M. Welgemoed, J. Bessant and D. Francis (2004) "Enabling Continuous Improvement: a Case Study Implementation", Journal of Manufacturing Technology Management, 15, No. 4, pp. 315324.

Japan Human Relations Association (1990) Kaizen Teian. Cambridge: Productivity Press.

JoRGEnSEN, F.; H. Boer and F. GerTSEN (2003) "Jump-starting Continuous Improvement Through self-assessment", International Journal of Operations \& Production Management, 23, No. 10, pp. 1260-1278.

JURAN, J. (1990) Juran y el Liderazgo para la Calidad. Un Manual para Directivos. Madrid, España: Editorial Díaz Santos.

KAYE, M. and R. ANDERSON (1999) "Continuous Improvement: The Ten Essential Criteria", International Journal of Quality \& Reliability Management, 16, No. 5, pp. 485-506.

LARAIA, A.C.; P. MOODY and R. HALL (1999) The Kaizen Blitz: Accelerating Breakthroughs in Productivity and Performance. New York: John Wiley \& Sons, Inc.

LEWIS, J. (2007) "Don't Lean on Kaizen Events", FDM, November, pp. 73-74.

LIKER, J. (2004) The Toyota Way: 14 Management Principles from the World's Greatest Manufacturer. New York: McGraw-Hill.

LILLRANK, P. (1995) "The transfer of Management Innovations from Japan", Organization Studies, 16, No. 6, pp. 971-989.

- and N. KANO (1989) Continuous Improvement-Quality Control Circles in Japanese Industry. Michigan: Center for Japanese Studies.

LINDBERG, P. and A. BERGER (1997) "Continuous Improvement: Design, Organization and Management", International Journal Technology Management, 14, No. 1, pp. 86-101.

MAGAÑA-CAMPOS, J. and E. ASPINWALL (2003) "Comparative Study of Western and Japanese Improvement Systems", Total Quality Management, 14, No. 4, pp. 423-436. 
MALLOCH, H. (1997) "Strategic and HRM aspects of kaizen: a case study", New Technology, Work and Employment, 12, No. 2, pp. 108-122.

MANOS, A. (2007) "The benefits of Kaizen and Kaizen events", Quality Progress, 40, No. 2, p. 47.

MARTINEZ-LORENTE, A.; F. DeWhURST and B.G. DALE (1998) "Total Quality Management: Origins and Evolution of the Term", The TQM Magazine, 10 , No. 5 , pp. $378-386$.

Melnyk, S.A.; R. Calanton, F.L. MONTABON and R.T. SMith (1998) "Shortterm action in pursuit of long-term improvements: introducing Kaizen Events", Production and Inventory Management Journal, 39, No. 4, pp. 69-76.

MONTABON, F. (2005) "Using Kaizen Events for Back Office Processes: Recruitment of frontline Supervisor Co-ops", Total Quality Management and Business Excellence, 16, No. 10, pp. 1139-1147.

Nemoto, M. (1987) Total Quality Control for Management. Strategies and Techniques from Toyota and Toyoda Gosei. New Jersey: Prentice Hall. Inc.

NEWITT, D.J. (1996) "Beyond BPR \& TQM - Managing through Processes: Is Kaizen Enough?", Industrial Engineering Conference Proceeding. London: Institution of Electric Engineers, Vol. 1, pp. 100-110.

Nikkei Sangyo Shimbun (2007) "En la búsqueda del Toyota Way por empresarios japoneses", septiembre (japonés).

OAKLAND, J.S. (1999) Total Organizational Excellence - Achieving World Class Performance. Oxford: Butterworth-Heinemann.

Ohno, T. (1978) Toyota Production System. Beyond Large-Scale Production. New York, NY: Productivity Press.

ORTíz, C. (2006) "The Kaizen 13", Industrial Engineer, April, pp. 33-34.

OSONO, E.; N. SHIMIZU and H. TAKEUCHI (2008) Extreme Toyota, Radical Contradictions that Drive Success at the World's best Manufacturer. New Jersey: John Wiley \& Songs.

PowELL, T.C. (1995) "Total Quality Management as Competitive Advantage: A Review and Empirical Study", Strategic Management Journal, 16, No. 1, pp. 15-37.

QUAZI, H.A.; J. JeMANGIN, L.W. KIT and C.L. KIAN (1998) "Critical Factors in Quality Management and Guidelines for Self-assesment, the Case of Singapore", Total Quality Management, 9, No. 1, pp. 35-55. 
RoBinson, A. (1990) Modern Approaches to Manufacturing Improvement. Portland, OR: Productivity Press.

SAVOLAINEN, T.I. (1999) "Cycles of Continuous Improvement. Realizing Competitive Advantage Through Quality", International Journal of Operations \& Production Management, 19, No. 11, pp. 1203-1222.

SaWAdA, N. (1995) "The Kaizen in Toyota Production System", material del curso de Company Wide Quality Control and Industrial Engineering. Nagoya: CHU-SAN-REN, Vol. 6, pp. 1-38.

SChroeder, D. and A. RoBinson (1991) "America's Most Successful Export to Japan-Continuous Improvement Programmes", Sloan Management Review, 32, No. 3, pp. 67-81.

SENGE, P. (1990) The Fifth Discipline. New York. N.Y: Double Day.

SHERIDAN, J. (1997) "Kaizen Blitz", Industry Week, 246, No. 16, pp. 19-27.

SPEAR, S. (2004) "Learning to Lead at Toyota", Harvard Business Review, 82 , No. 5 , pp. $78-86$.

StRATEGIC DiRECTION (2004) "Kaizen at Nippon: Behind the Theory", Strategic Direction, 20, No. 5, pp. 23-25.

STYHRE, A. (2001) "Kaizen, Ethics, and Care of the Operations: Management after Empowerment", Journal of Management Studies, 38, No. 6, pp. 795-810.

SUÁREZ-BARRAZA, M.F. (2001) "La filosofía del Kaizen, una aplicación práctica en un área de servicio del sector público", Revista CONTACTO. La revista de la Calidad Total, 11, pp. 11-16.

- (2007) El KAIZEN: La filosofía de Mejora Continua e Innovación Incremental detrás de la Administración por Calidad Total. México, D.F.: Panorama.

-, E. Bou and C. CATAldo (2008) "Finding Standards, Routines and NonRoutines in Toyota Production System (TPS): Standardization without Standardization?", Lean Manufacturing Journal-Reliable Plant Magazine, September, 7, pp. 1-32.

- and J. RAmIS-Pujol (2008) "Aplicación y evolución de la Mejora Continua de Procesos en la Administración Pública", Journal Globalization, Competitiveness \& Governability GCG Georgetown University- Universia, 2, No. 1, pp. 74-86.

SVENSSON, G. (2006) "Sustainable Quality Management: a Strategic Perspective", The TQM Magazine, 18, No. 1, pp. 22-29. 
TAKEYUKI, F. (1995) "Implementing C-JIT (New JIT) A Practical Approach", Material del curso de Company Wide Quality Control and Industrial Engineering. Nagoya: CHU-SAN-REN, 8, pp. 1-38.

TANNER, C. and R. RONCARTI (1994) "Kaizen Leads to Breakthroughs in Responsiveness and the Shingo Prize at Critikon", National Productivity Review, 13, No. 4, pp. 517-531.

TILLINGHURST, D. (1997) "Kaizen Blitz", Industry Week, 246, No. 19, pp. 1927.

TOZAWA, B. and N. BODEK (2002) Kaizen Rápido y Fácil. Madrid: TGP Hoshin.

Vonk, J. (2005) "Process Improvement in Business Permits through Kaizen", Innovations Management, Spring, pp. 33-34.

WENNECKE, G. (2008) "Kaizen-Lean in a week: how to implement improvements in healthcare settings in a week", Medical Laboratory Observer, August, pp. 28-31.

WitTEnBerG, G. (1994) "Kaizen, The Many Ways of Getting Better", Assembly Automation, 14, No. 4, pp. 12-17.

WOMACK, J.P.; D.T. JONES and D. TOSS (1990) The Machine that Changed the World. New York, N.Y.: Rawson, Associates. 